\title{
Evaluation of the pulmonary valve and right ventricular outflow tract in the prone position during invasive mechanical ventilation - Is it possible?
}

Cesar Del Castillo Gordillo ${ }^{1}$, Francisca Yañez Vidal ${ }^{2}$, Marcelo Luque Gonzalez ${ }^{2}$, Mario

Alfaro Diaz ${ }^{2}$, and Arnulfo Begazo Gonzales ${ }^{1}$

${ }^{1}$ Hospital DIPRECA

${ }^{2}$ Hospital Clínico San Borja Arriarán

November 16, 2020

\begin{abstract}
In the COVID-19 pandemic, we performed a series of echocardiograms using subcostal views. After placing a patient in prone position during invasive mechanical ventilation, the echocardiogram transducer was placed under the patient in the left subcostal position. This performance allowed us to evaluate the function of the pulmonary valve and estimate pulmonary pressure. This is a complement to the monitoring with transthoracic echocardiogram in prone position.
\end{abstract}

\section{Title page}

Evaluation of the pulmonary valve and right ventricular outflow tract in the prone position during invasive mechanical ventilation - Is it possible?

Authors: César Del Castillo Gordillo MD ${ }^{1,2}$, Francisca Yañez Vidal MD ${ }^{2}$, Marcelo Luque Gonzalez $\mathrm{MD}^{2}$, Mario Alfaro Diaz MD ${ }^{2}$, Arnulfo Begazo Gonzales MD ${ }^{1}$.

${ }^{1}$ Cardiovascular center, DIPRECA Hospital.

${ }^{2}$ Cardiovascular center, San Borja Arriaran Clinic Hospital.

Correspondending author: César Del Castillo Gordillo MD, Cardiologist, Cardiovascular center, DIPRECA Hospital. 1200 Vital Apoquindo, 7601003, Santiago, Chile. Email:cesardelcastillogor@gmail.com.

Author contributions: CDCG and FYV: case identification, image editing, writing and research. MAD and ABG: Image editing and final proof reading.

Abstract: In the COVID-19 pandemic, we performed a series of echocardiograms using subcostal views. After placing a patient in prone position during invasive mechanical ventilation, the echocardiogram transducer was placed under the patient in the left subcostal position. This performance allowed us to evaluate the function of the pulmonary valve and estimate pulmonary pressure. This is a complement to the monitoring with transthoracic echocardiogram in prone position.

Keywords: Cardiology, Echocardiography, 2D Echocardiography, Doppler echocardiography, Pulmonary valve, Pulmonary hypertension.

\section{Description:}


During the COVID-19 pandemic, we performed a series of transthoracic echocardiograms (TTE) using subcostal view and observed that the evaluation of the pulmonary valve (PV) and the right ventricular outflow tract (RVOT) was possible in prone position $(\mathrm{PP}) .{ }^{1}$

After placing in PP during invasive mechanical ventilation (IMV), the patient's left arm was extended overhead, and a pillow was placed only under the left hemithorax to elevate it and facilitate subcostal view (Figure 1). The transducer was placed under the patient, in the left subcostal position, and an orientation index marker pointing towards the patient's left shoulder was used to observe the four cardiac chambers (Figure 1B). Subsequent subtle upward movement of the transducer tail permitted us to view the PV and a small portion of the RVOT (Figure 1C, 2A and 2B). A further counter-clockwise rotation of the transducer allowed us to view the short axis of the RVOT (Figure 1D and 2C). This approach allowed us to observe the opening of the PV by 2D visualization and the transpulmonary flow by colour Doppler (Figure 2D). Continuous Doppler allowed us to calculate the gradients of the PV (Figure 2E), and pulsed Doppler at RVOT level helped us estimate the pulmonary pressure based on the shape of its curve and by measuring the acceleration time (Figure 2F).

TTE has emerged as an alternative for monitoring patients in the PP during IMV, with adequate imaging achieved because of a proximity of the heart to the chest wall in PP. ${ }^{2}$ Previous studies have reported that apical views allow evaluation of ventricular and valve function and that of the inferior vena cava. ${ }^{3}$ Recently, a study has reported the performance of a subcostal view examination in PP and briefly described the PV. ${ }^{1}$ However, it is not described a detailed evaluation of the PV or RVOT.

Our technique has the advantage of allowing evaluation of the PV function and the presence of pulmonary hypertension. ${ }^{4}$ This is a novel possibility to perform TTE in the PP, where we could discard the PV disease and evaluate the pulmonary pressure by a different approach.

\section{References:}

1. Del Castillo Gordillo C, Yañez Vidal F, Begazo Gonzales A, Alfaro Diaz M. Description of the position and performance of an echocardiogram by subcostal view during the prone position in a patient with severe pneumonia caused by COVID-19. BMJ Case Rep 2020;13:e239230. doi:10.1136/bcr-2020-239230.

2. Chino JP, Marks LB. Prone positioning causes the heart to be displaced anteriorly within the thorax: implications for breast cancer treatment. Int J Radiat Oncol Biol Phys 2008;70:916-20. doi: 10.1016/j.ijrobp.2007.11.001.

3. Gibson LE, Di Fenza R, Berra L, et al. Transthoracic echocardiography in prone patients with acute respiratory distress syndrome: a feasibility study. Crit Care Explor 2020;2:e0179. DOI: 10.1097/CCE.0000000000000179.

4. Parasuraman S, Walker S, Loudon BL, et al. Assessment of pulmonary artery pressure by echocardiography-A comprehensive review. Int J Cardiol Heart Vasc 2016;12:45-51. doi: 10.1016/j.ijcha.2016.05.011.

\section{Figure legends:}

Figure 1. How to perform the evaluation of pulmonary valve and right ventricular outflow tract: A, position to perform echocardiogram by subcostal view in prone position, with a pillow placed under left hemithorax (light blue). B, the echocardiogram transducer was placed under the patient in the left subcostal position and orientation index marker pointing towards the patient's left shoulder to view the 4 cardiac chambers. $\mathrm{C}$, subtle upward movement of the transducer tail to view the pulmonary valve and a small portion of the RVOT. D, counter-clockwise rotation of the transducer to view the short axis of the RVOT. Abbreviations A: Aorta. AV: aortic valve. LA: left atrium. LV: left ventricle. PA: pulmonary artery. PV: pulmonary valve. RA: right atrium. RV: right ventricle. RVOT: right ventricle outflow tract. Illustration by César del Castillo Gordillo.

Figure 2. Images obtained: A, visualization of pulmonary valve and right ventricular outflow tract. B, continuous Doppler of Pulmonary valve. C, short axis of the right ventricular outflow tract. D, colour Doppler 
of short axis of the right ventricular outflow tract. E, Continuous Doppler of Pulmonary valve. F, Pulsed Doppler at right ventricular outflow tract level.

\section{Hosted file}

FIGURE 1.pdf available at https://authorea.com/users/376089/articles/493182-evaluation-ofthe-pulmonary-valve-and-right-ventricular-outflow-tract-in-the-prone-position-duringinvasive-mechanical-ventilation-is-it-possible

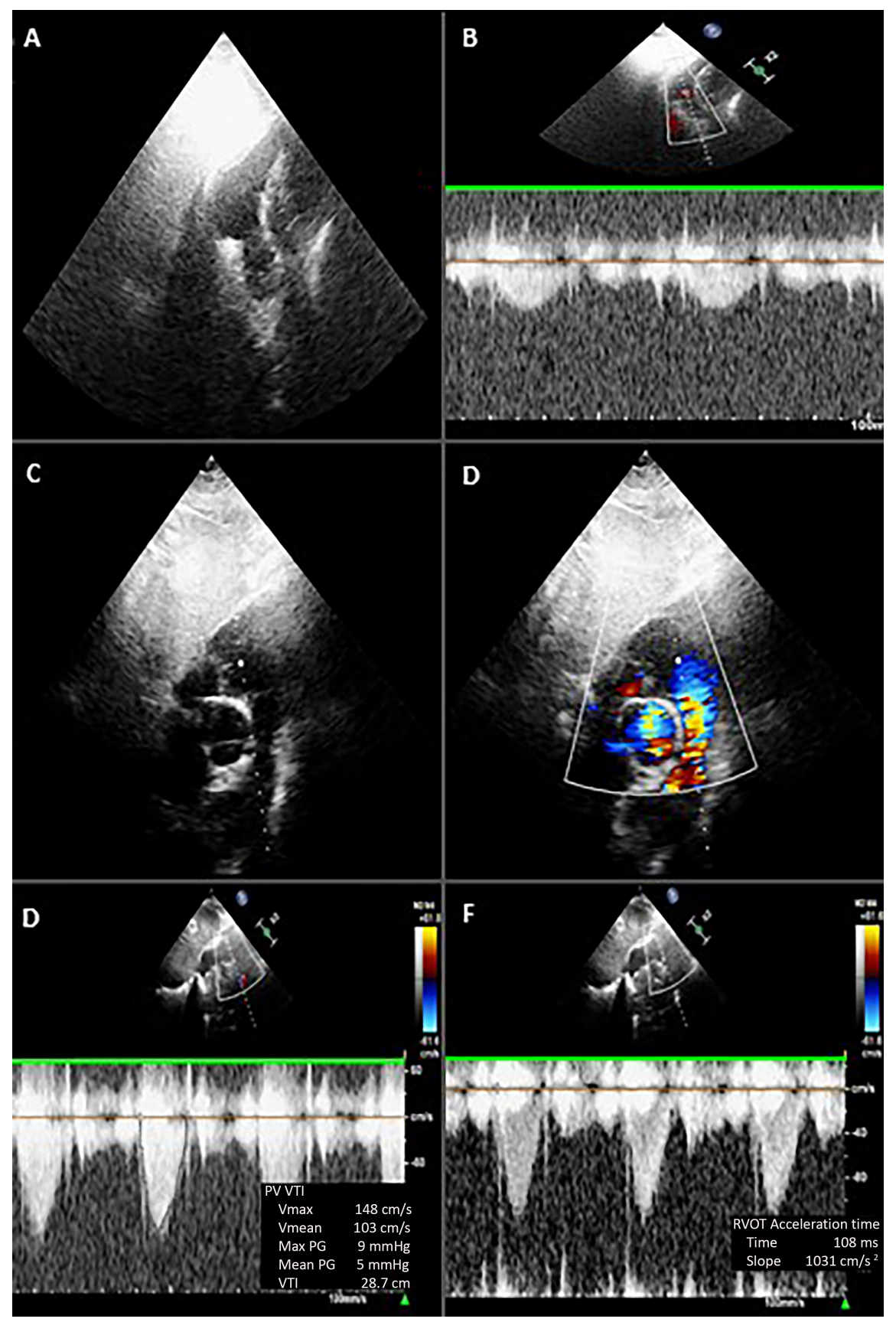

